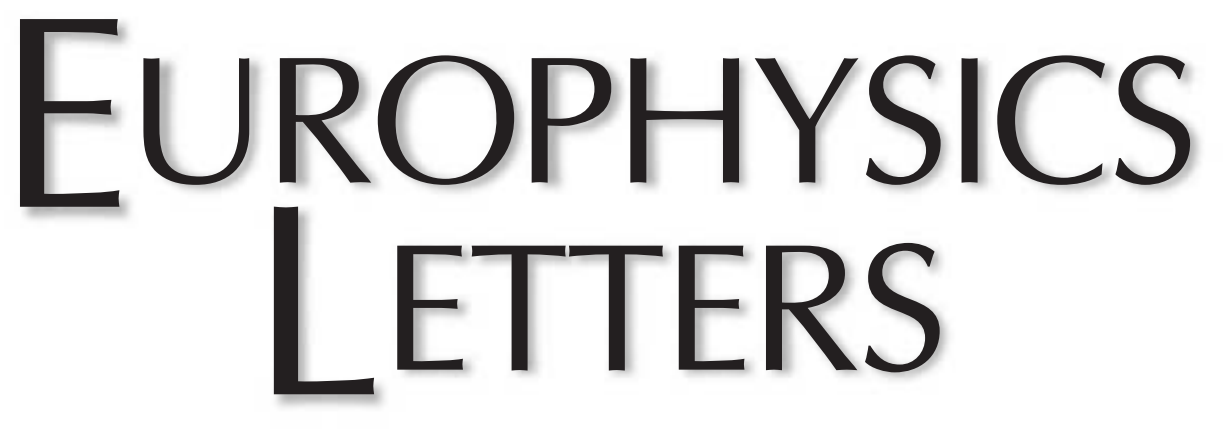

OFFPRINT

Vol. $62 \bullet$ Number $6 \bullet$ pp. 897-903

Buckling instability induced by polymer solution drying $* * *$

L. Pauchard and C. Allain
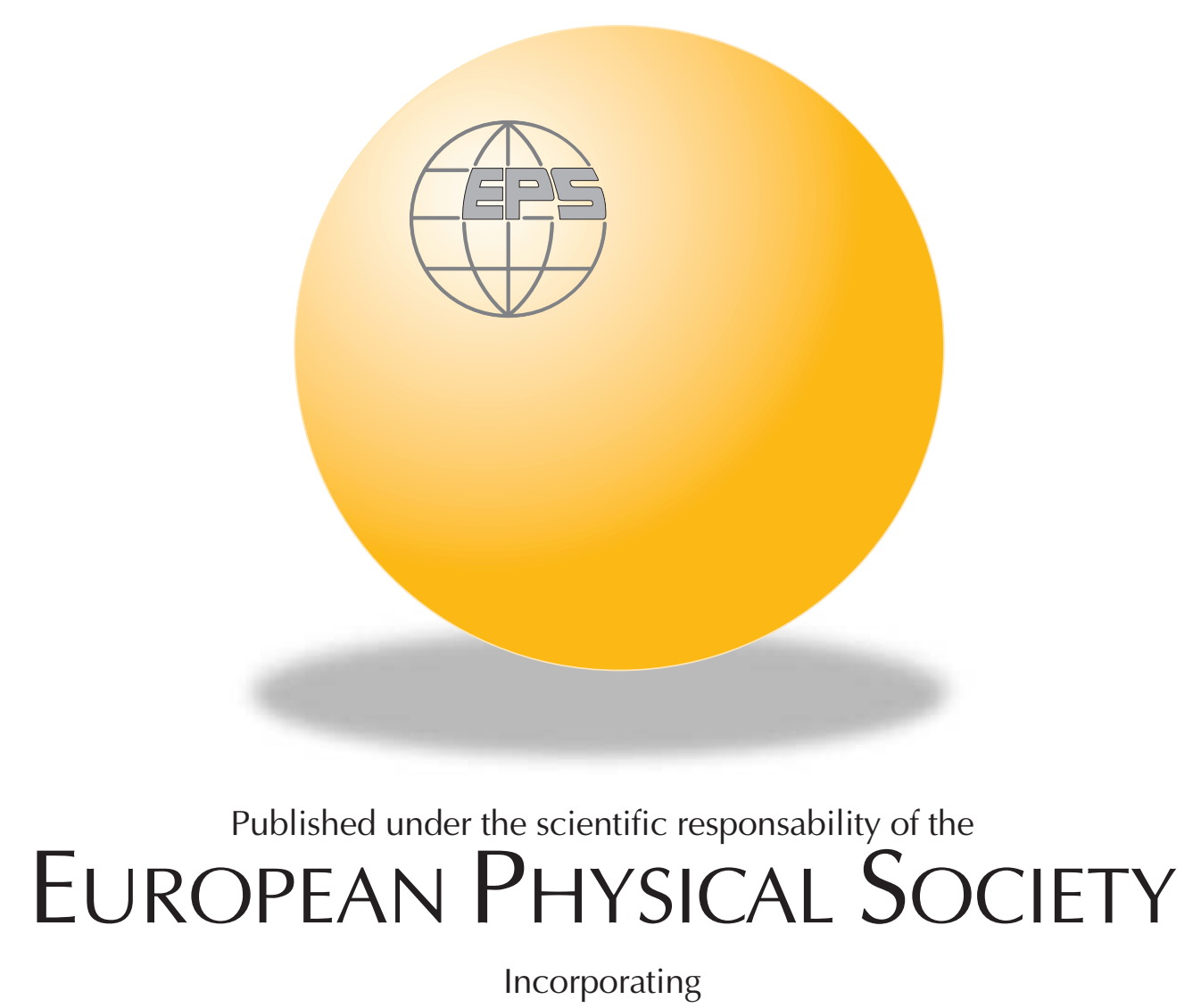

JOURNAL DE PhySIQUE LETTRES • LETTERE AL NUOVO CIMENTO 


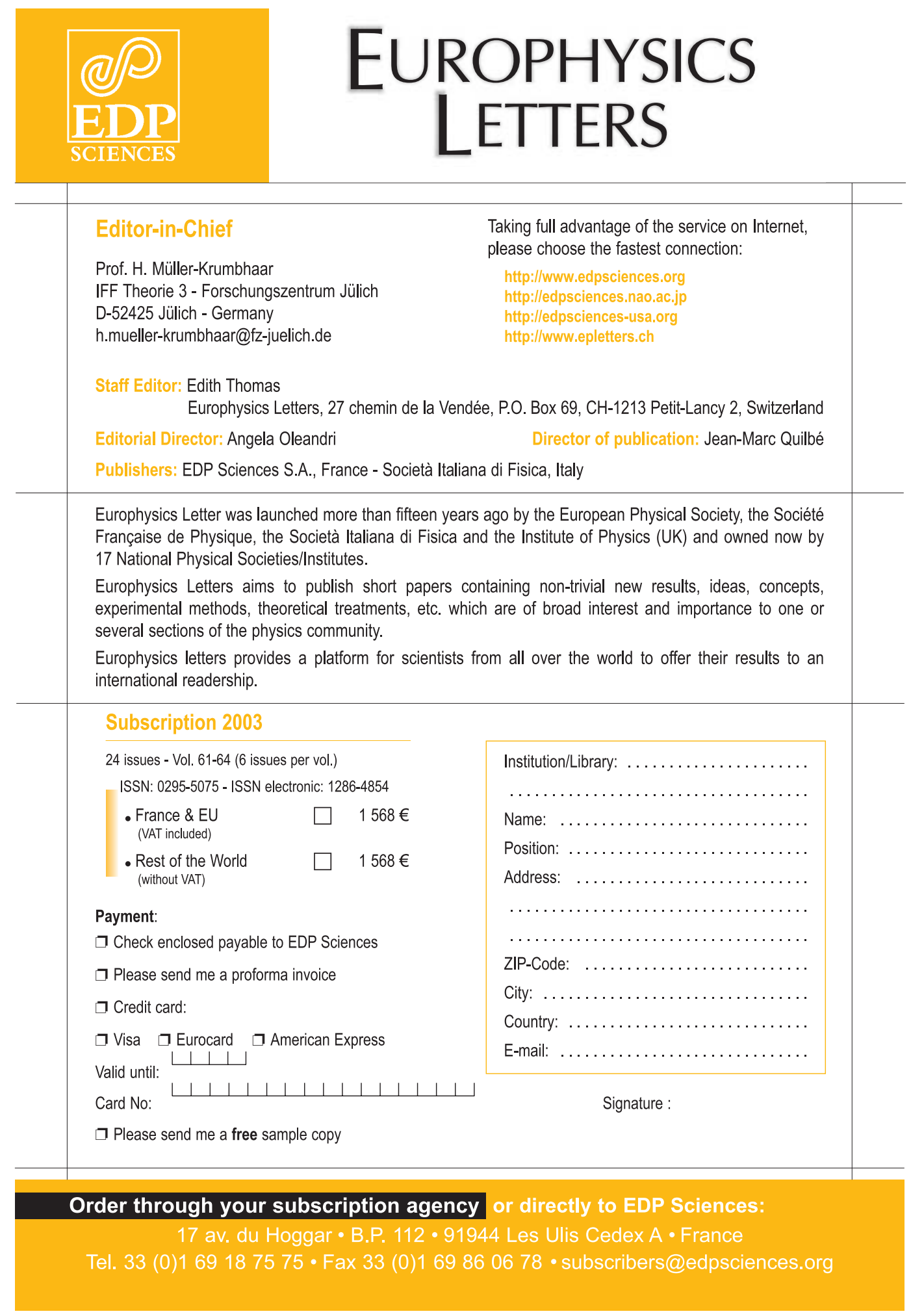


Europhys. Lett., 62 (6), pp. 897-903 (2003)

\title{
Buckling instability induced by polymer solution drying
}

\author{
L. Pauchard and C. Allain \\ Laboratoire FAST, Bât. 502, Campus Universitaire - 91405 Orsay Cedex, France
}

(received 19 August 2002; accepted in final form 22 April 2003)

PACS. 83.80.Rs - Polymer solutions.

PACS. 46.32.+x - Static buckling and instability.

PACS. 64.70.Pf - Glass transitions.

\begin{abstract}
The large shape distortions that occur during the drying of sessile drops of polymer solution are shown to be related to a buckling instability. As solvent evaporates, polymers accumulate near the vapor/drop interface and, depending on the experimental conditions, can form a glassy skin which bends as the volume it encloses decreases. A comparison of the times that characterize drying kinetics and glassy skin formation enables us to predict instability occurrence. Good agreement is found with measurements performed at different polymer concentrations, drop volumes and drying rates.
\end{abstract}

The drying of complex fluids such as polymer solutions and colloidal dispersions involves complex spatial and temporal evolutions which are related to a large number of microscopic phenomena like solvent diffusion, transfers at the vapor/medium interface, gelation or glass transition... In particular, an increase of the concentration of the non-volatile components modifies the rheological properties of the medium which, initially fluid, can change into a viscoelastic or brittle solid. In practice, these phenomena are crucial for coating technology since they give rise to major defects such as cracks, debonding and wrinkles, thus conditioning the final film quality. From the fundamental standpoint, interest has grown recently in surface instabilities and crack patterns arising from shrinkage [1-6].

As polymer solutions dry, high-concentration gradients form near the vapor/solution interface [7]. Depending on the physicochemical properties of the system concerned, the outer layer, with the higher polymer concentration in, can undergo a glass transition. We show here that the formation of this glassy skin is the cause of a buckling instability. In fact if the outer layer becomes glassy, it behaves as an elastic shell which, however, does not impede evaporation. This shell bends as the volume it encloses decreases, while its surface remains constant.

In the present letter, we consider the geometry of a drop deposited on a flat substrate (see fig. 1). As recently investigated for colloidal suspensions [8,9], a key difference is observed between the drying of a polymer solution drop and of a pure solvent drop. In fact, as for colloids, polymer deposition and adhesion onto the substrate leads to a strong pinning of the three-phase line: the drop evaporates with a constant contact area with the substrate, contrary to a pure solvent drop, which recedes with a constant contact angle. Depending on the polymer concentration, two different drop shape evolutions are observed. At low concentrations (see fig. 1a), the drop progressively flattens with solvent evaporation and the apex height steadily decreases. In the final stage, a flat "pancake" forms, with a depressed central zone for the lowest concentrations. On the contrary, if the concentration is high

(c) EDP Sciences 

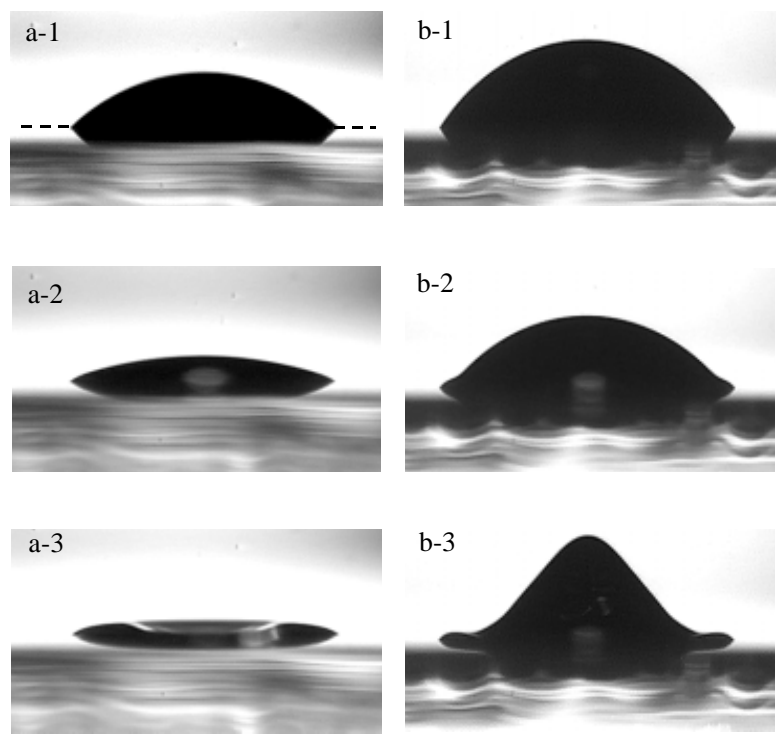

Fig. 1 - Lateral views showing stable (a) and unstable (b) evolutions of Dextran solution drops deposited on a glass slide. (a) Polymer concentration $\omega_{\mathrm{p}}=0.20 \mathrm{~g} / \mathrm{g}$ : (a-1) just after deposition; the dotted line corresponds to the drop/substrate contact, the drop base diameter is $4 \mathrm{~mm}$, it remains constant during drying. (a-2) 6 minutes after deposition: the drop steadily flattens. (a-3) End of drying: the drop forms a flat "pancake". (b) Polymer concentration $\omega_{\mathrm{p}}=0.40 \mathrm{~g} / \mathrm{g}$ : (b-1) just after deposition. (b-2) 6 minutes after deposition: just before instability beginning. (b-3) 7 minutes after deposition: the apex height has increased and reaches a peak leading to the typical "Mexican hat" shape at the end of drying.

enough, the drop shape is severely distorted (see fig. 1b). After a steady decrease, the apex height starts to decrease and can reach a value greater than its initial value. In the final stage, the drop shows the shape of a "Mexican hat". We investigated the occurrence of this shape instability: the characteristic time when the drop surface begins to distort was measured as a function of polymer concentration, drop volume and drying rate. The estimation of the polymer concentration near the vapor/drop interface and the conditions for glass formation allow calculating the conditions for instability occurrence. Good agreement is found with the experimental results.

The experiments were performed using concentrated solutions of a water-soluble polysaccharide, Dextran (Sigma Aldrich Chemical Company). Two molecular weights (77000 and $37500 \mathrm{~g} / \mathrm{mol}$ ) were used; no difference was found between the two samples. Solutions were prepared by dissolving a given quantity of polymer in water (quality Milli- $\rho$ ). Surface tension measurements showed a decrease with increasing polymer concentration (the relative variation is $\cong-10 \%$ between water and $0.1 \mathrm{~g} / \mathrm{g}$ solution). The glass transition of our polymer samples, determined by DSC (Differential Scanning Calorimetry), was found to occur at the temperature of $220^{\circ} \mathrm{C}$. The glass transition temperature for a polymer solution is lower than that of the pure polymer and decreases when the polymer concentration $\omega_{\mathrm{p}}$ decreases [10]. Thus, at a given temperature $T_{\exp }$, the solution has a lower glass transition temperature than $T_{\exp }$ for low polymer concentration and a higher glass transition temperature than $T_{\exp }$ for high polymer concentration. Hence a concentration $\omega_{\text {pg }}$ exists such that the solution is fluid when 

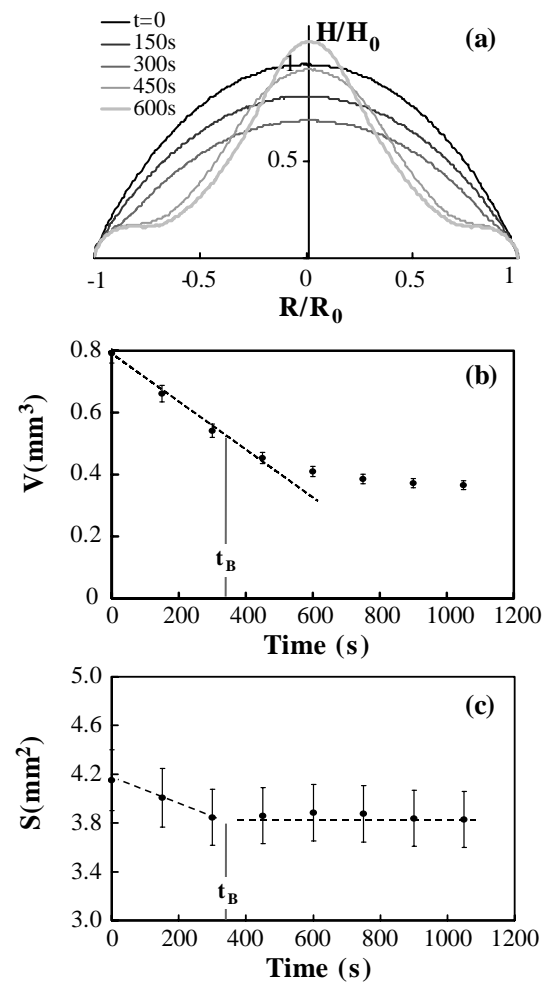

Fig. 2 - Time evolution of a drop of polymer concentration $\omega_{\mathrm{p}}=0.40 \mathrm{~g} / \mathrm{g}$ : (a) dimensionless profiles recorded at different times. (b) and (c) are, respectively, the variations in volume and surface area vs. time. We have plotted the buckling characteristic time $t_{\mathrm{B}}$ at which the instability starts.

$\omega_{\mathrm{p}}<\omega_{\mathrm{pg}}$ and glassy when $\omega_{\mathrm{p}}>\omega_{\mathrm{pg}}$. During solvent evaporation, the polymer concentration increases, and the initially fluid solution becomes glassy.

In this work, we selected the geometry of sessile drops as ideal for experimental studies. This geometry allows accurate measurements and easy investigation of the influence of the various parameters. The solution drops are deposited onto horizontal slides using micropipettes. The glass slides are carefully cleaned before use to allow fairly good contact angle reproducibility: $\theta_{0}=45^{\circ} \pm 4^{\circ}$. The change in shape is examined with a set-up that allows accurate observations: both lateral and top views are recorded and special care is taken to avoid optical distortions. The set-up is placed inside a glove box in which the relative humidity, $R H$, is controlled within $\pm 5 \%$ in relative value $\left(R H=n_{\mathrm{w} \infty} / n_{\text {wsat }}\right.$, where $n_{\mathrm{w} \infty}$ and $n_{\text {wsat }}$ are the water concentrations in air at infinity and at saturation). The first profile measured just after drop deposition is used to determine the initial drop characteristics: $R_{0}$ the radius of the contact base, $\theta_{0}$ the contact angle and $H_{0}$ the apex height. Profiles measured at different times are normalized by the contact base radius and by the initial apex height. For each drop, the axisymmetry is checked to ensure that the contact base is circular (using top views) and that the profiles are symmetrical. We can then calculate the volume $V$ and the vapor/drop surface area $S$ at different times from the profile. Spatio-temporal diagrams are also constructed to determine the variations in apex height with time.

Let us first consider a typical unstable case: for example, a $5 \mathrm{~mm}^{3}$ drop of $0.40 \mathrm{~g} / \mathrm{g}$ solution. The profiles and volume and surface area variations with time are shown in fig. 2 . In the first 


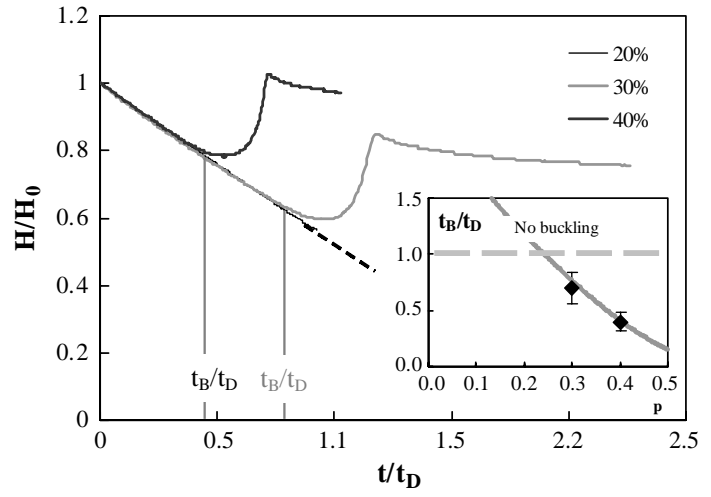

Fig. 3

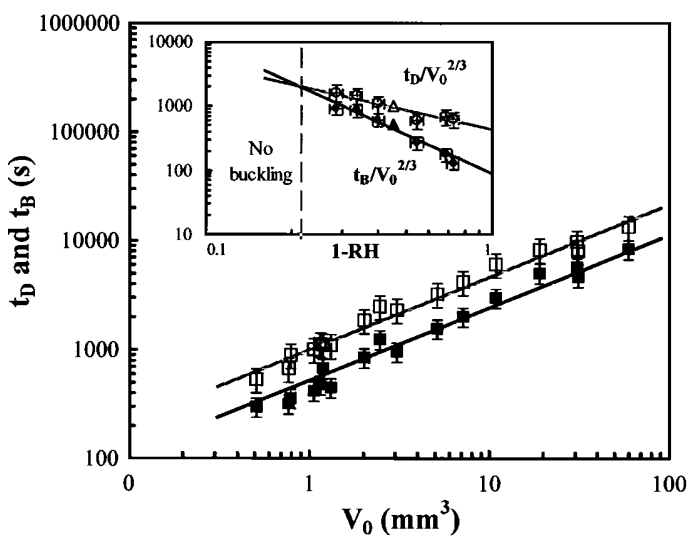

Fig. 4

Fig. 3 - Dimensionless variations of apex height $\left(H / H_{0}\right) v s$. time $\left(t / t_{\mathrm{D}}\right)$ at 3 polymer concentrations. The buckling time $t_{\mathrm{B}}$ is defined as the time at which the decrease in $H / H_{0}$ begins to slow down. At the lowest concentration, the experimental values are continued by an eye guide (dashed line). Inset: variations of $t_{\mathrm{B}} / t_{\mathrm{D}}$ vs. $\omega_{\mathrm{p}}$ and corresponding theoretical prediction (solid line).

Fig. 4 - log-log plot of variations of the drying time $(\square)$ and buckling time ( $\mathbf{\square})$ as a function of initial drop volume $(R H=55 \%)$. Inset: log-log plot of $t_{\mathrm{D}} / V_{0}{ }^{2 / 3}$ (०) and $t_{\mathrm{D}} / V_{0}{ }^{2 / 3}(\bullet)$ vs. $1-R H$. The triangles correspond to $R H=55 \%$. All solid lines correspond to theoretical predictions.

stage, the drop progressively flattens: its apex height decreases steadily and its shape is not substantially modified. During the same time interval, the volume decreases linearly with time (fig. 2b) as well as the surface area (fig. 2c). After the beginning of instability, which, in the present case, takes place between 300 and $450 \mathrm{~s}$, the profiles are strongly distorted: the apex height increases. The surface area remains constant while the volume keeps decreasing but much more slowly. This is related to the increase in polymer concentration at the drop surface: when it reaches $\omega_{\mathrm{pg}}$, a glassy skin forms which is too thin to significantly change the evaporation rate but which bends as the volume it encloses decreases. Later, under the skin concentration and thickness increase, the evaporation rate slows down. A simple way to qualitatively identify the existence of this skin is to suck up the drop using a micro-pipette. If the test is performed during the first stage of drying, no solid skin is observed in the central part of the drop, which can be entirely sucked up (only a solid ring along the threephase line remains on the substrate). On the contrary, no solution can be sucked up after the instability beginning. This simple test shows that the instability observed is associated with the formation of a solid skin at the drop surface. Note that any subsequent change in shape cannot be due to a wetting instability, since the surface tension decreases with polymer concentration and the surface tension of the drop outer layer is therefore lower than that of the drop core solution [11]. Moreover, during this stage, the surface area remains constant (see fig. 2c). Once formed, the glassy polymer skin behaves as an elastic shell, but without impeding evaporation; with solvent loss, the confined volume decreases leading to a buckling instability.

To quantitatively describe the drop shape changes, two characteristic times are introduced. The first is the drying time $t_{\mathrm{D}}$, which describes the rate of volume variation: $t_{0}{ }^{-1}=$ $-\frac{1}{V_{0}}\left(\frac{\partial V}{\partial t}\right)_{t=0}$. Time $t_{\mathrm{D}}$ gives an order of magnitude of the time needed for the drop to dry and therefore depends on the humidity, drop size and contact angle. In practice, given the contact angle dispersion, the accuracy on $t_{\mathrm{D}}$ is $\pm 25 \%$. The second time introduced is the buckling 
time $t_{\mathrm{B}}$, which characterizes the beginning of the instability. Time $t_{\mathrm{B}}$ is determined from the spatio-temporal diagrams. Figure 3 shows the variations of the apex height with time obtained for three polymer concentrations. For $\omega_{\mathrm{p}}=0.20 \mathrm{~g} / \mathrm{g}$, the apex height decreases steadily with time: no instability takes place. At the highest concentrations, $\omega_{\mathrm{p}}=0.30$ and $0.40 \mathrm{~g} / \mathrm{g}$, after a decrease similar to the one observed for $\omega_{\mathrm{p}}=0.20 \mathrm{~g} / \mathrm{g}, H / H_{0}$ starts increasing and, later, after a steep increase can reach a value that can exceed 1 . Time $t_{\mathrm{B}}$ is defined as the time at which the decrease of $H / H_{0} v s$. time begins to differ from the regular decrease, i.e. $t_{\mathrm{B}}$ corresponds to the beginning of the instability. In practice, the accuracy on $t_{\mathrm{B}}$ determination is $\pm 20 \%$. In the case of fig. $2, t_{\mathrm{B}}=360 \mathrm{~s}$, in agreement with volume and surface area variations. Systematic measurements performed by varying $V_{0}$ and $R H$ are displayed in fig. 4 .

To interpret these results and model the instability occurrence conditions, let us derive the expressions of $t_{\mathrm{D}}$ and $t_{\mathrm{B}}$. In our experimental conditions, the transfer of water in air is limited by diffusion. Thus, as long as it is the dominant mechanism, the evaporation rate $W_{\mathrm{E}}=-(1 / S)(\mathrm{d} V / \mathrm{d} t)$ is given by $[12]$

$$
W_{\mathrm{E}}=A\left(\theta_{0}\right)\left[\left(n_{\mathrm{wsatp}}-n_{\mathrm{w} \infty}\right) / n_{\mathrm{l}}\right] D_{\mathrm{w}} / R_{0},
$$

where $A\left(\theta_{0}\right)$ is a numerical factor, $n_{1}$ is the number of moles per unit volume in liquid water and $D_{\mathrm{w}}$ the diffusion coefficient of water into air. For a polymer solution $n_{\text {wsatp }}$ is smaller than $n_{\text {wsat }}$ (for pure water) and can be expressed using the Flory-Huggins equation: $n_{\text {wsatp }} / n_{\text {wsat }}=\left(1-\Phi_{\mathrm{p}}\right) \times \exp \left[\Phi_{\mathrm{p}}+\chi \Phi_{\mathrm{p}}{ }^{2}\right] ; \Phi_{\mathrm{p}}$ is the polymer volume fraction [13]. For aqueous Dextran solutions the $\chi$-parameter, which characterizes the affinity between the solvent and the polymer, is $\chi \cong 0.5[14,15]$; so that an increase in $\omega_{\mathrm{p}}$ from 0.40 to $0.60 \mathrm{~g} / \mathrm{g}$ leads to a decrease of $n_{\text {wsatp }} / n_{\text {wsat }}$ from 0.99 to 0.94 . This small variation explains why the evaporation rate remains almost constant during the first drying stage. Time $t_{\mathrm{D}}$ is related to the initial evaporation rate $W_{\mathrm{E} 0}$ by $t_{\mathrm{D}}{ }^{-1}=\left(S_{0} / V_{0}\right) W_{\mathrm{E} 0}$. Hence, for drops of same contact angle and concentration, $t_{\mathrm{D}}$ is expected to vary as

$$
t_{\mathrm{D}} \sim(1-R H)^{-1} V_{0}^{2 / 3} .
$$

As shown in fig. 4 , the experimental values of $t_{\mathrm{D}}$ are in good agreement with the prediction. In addition, knowing the values of the different quantities involved in (1) $[12,16]$, we can calculate the prefactor in eq. (2). It is found to be $450 \mathrm{~s} / \mathrm{mm}^{2}$, which agrees with the results obtained by varying $V_{0}$ or $R H$ (see fig. 4 ).

To calculate the buckling time $t_{\mathrm{B}}$, we must account for transfers inside the solution. In fact, as evaporation proceeds, the polymer concentration at the vapor/drop surface increases and high concentration gradients are formed. Solvent flux conservation at air drop interface writes: $W_{\mathrm{E}}=D_{\mathrm{m}} \nabla \varphi_{\mathrm{p}}$, where $D_{\mathrm{m}}$ is the mutual diffusion coefficient and $\varphi_{\mathrm{p}}$ the local value of the polymer volume fraction. For most polymer/solvent systems, $D_{\mathrm{m}}$ has been found to range between $10^{-10}$ and $10^{-9} \mathrm{~m}^{2} / \mathrm{s}$ [17]. In order of magnitude, $\nabla \varphi_{\mathrm{p}}$ expresses as $\nabla \varphi_{\mathrm{p}} \cong$ $\left(\varphi_{\mathrm{ps}}-\varphi_{\mathrm{p} 0}\right) / \sqrt{D_{\mathrm{m}} t}$, where $\varphi_{\mathrm{ps}}$ and $\varphi_{\mathrm{p} 0}$ are, respectively, the polymer volume fraction at the vapor/drop surface and in the core of the drop. Using $\varphi_{\mathrm{p} 0}$ assumes that the diffusion length, $\sqrt{D_{\mathrm{m}} t}$, remains smaller than the drop height: this is always satisfied for drops having large contact angles as investigated here. Since $W_{\mathrm{E}}$ is constant during the first drying stage, $\varphi_{\mathrm{ps}}$ can be expressed as a function of time. Then, assuming that the instability starts when $\varphi_{\mathrm{ps}}=\varphi_{\mathrm{pg}}$, we find

$$
t_{\mathrm{B}}=\frac{D_{\mathrm{m}}\left(\varphi_{\mathrm{pg}}-\varphi_{\mathrm{p} 0}\right)^{2}}{W_{\mathrm{E} 0}{ }^{2}} .
$$

For drops of same contact angle and concentration, $t_{\mathrm{B}}$ is found to vary as

$$
t_{\mathrm{B}} \sim(1-R H)^{-2} V_{0}^{2 / 3} .
$$




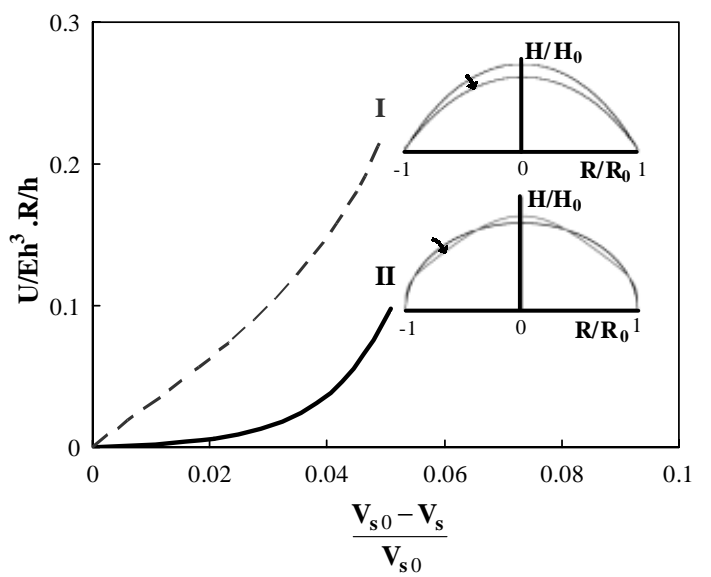

Fig. 5 - Dimensionless elastic energy $\left(U / E h^{3} \cdot R / h\right)$ vs. relative volume $\left(\left(V_{\mathrm{s} 0}-V_{\mathrm{s}}\right) / V_{\mathrm{s} 0}\right)$ in the case of two configurations: elastic shell flattening (I) and sinusoidal perturbation around a spherical cap shape (II). $E$ is Young's modulus, $h$ the shell thickness (assumed to be uniform and constant over time) and $R$ the radius of curvature characteristic of the initial spherical cap shape. $V_{\mathrm{s} 0}$ denotes the drop volume when the skin forms at the surface of the drop, $V_{\mathrm{s}}$ denotes the volume variation during buckling of the elastic shell. (The energy reference for a non-deformed shell is assumed to be 0).

Note that a complete calculation would also consider that the stress on the skin has to overcome a critical value to lead to the instability [18]. In practice, the stress generated by the volume decrease under solvent loss increases rapidly, and can be assumed to exceed the buckling critical value almost as soon as the skin is formed.

To understand the formation of the "Mexican hat" shape, we estimated the elastic energy for a thin elastic shell under the decrease of its inner volume. The energy of a spherical cap shape with hinge supports at the boundary, continuously flattening under the decrease of its inner volume, is denoted configuration I [19] (see fig. 5). We investigated a sinusoidal perturbation around a spherical cap shape which induces an axisymmetric change of curvature; this is called configuration II (see ref. [20]). The energy of configuration I arises as a balance between two terms: the bending energy (concentrated near the circumferential boundary) and the stretching energy due to the compression of the spherical cap region, found to be $10 \%$ higher than the former. The energy of contribution II is simply due to a bending effect concentrated in the axisymmetric circular fold. Comparison of the energies of both configurations shows configuration II to be energetically favorable (fig. 5). Our experimental results are also well adjusted by this configuration.

The influence of humidity on the buckling instability was also studied; indeed varying $R H$ changes the drying rate and hence the skin thickness and drying stresses [21]. In our experimental conditions no change was observed in the $t_{\mathrm{B}}$ variations, showing that the instability is governed by glass skin formation. A rough estimate can be made of the prefactor involved in (4), assuming $D_{\mathrm{m}}=4 \times 10^{-10} \mathrm{~m}^{2} / \mathrm{s}$ and $\varphi_{\mathrm{pg}}=0.5\left(\omega_{\mathrm{pg}} \cong 0.62 \mathrm{~g} / \mathrm{g}\right)$, it is $\cong 100 \mathrm{~s} / \mathrm{mm}^{2}$. As shown in figs. 3 , inset, and 4 , good agreement is found with all the experimental results.

Comparing the two characteristic times $t_{\mathrm{B}}$ and $t_{\mathrm{D}}$ allows to determine the condition for buckling instability occurrence. If $t_{\mathrm{B}} \gg t_{\mathrm{D}}$, the drop dries before buckling has had time to begin, while if $t_{\mathrm{B}} \ll t_{\mathrm{D}}$, instability occurs during drying. The ratio $t_{\mathrm{B}} / t_{\mathrm{D}}=\left(S_{0} / V_{0}\right) D_{\mathrm{m}}\left(\varphi_{\mathrm{pg}}-\right.$ $\left.\varphi_{\mathrm{p} 0}\right)^{2} / W_{\mathrm{E} 0}$ is independent of drop volume and decreases with increasing concentration, in good agreement with figs. 4 and 3 , inset. 
In conclusion, we have demonstrated that the shape instability affecting concentrated polymer solution drops under drying is a buckling instability due to the formation of a glassy skin at the vapor/solution interface. Measurements of the times characterizing solvent evaporation and incipient instability show good agreement with a model that takes account of solvent transfers and increasing polymer concentration at the drop surface. Comparing these two times helps to predict the conditions for instability occurrence. Further experiments on the influence of the different parameters and of the geometry will help to test the universality of our model and the possible existence of secondary instabilities.

The authors are grateful to P. G. DE Gennes and L. TAlini for their interest in the subject and thank T. GIRARD, F. PARISSE and G. CALligari for their contributions to the experiments. Laboratory "Fluides, Automatique et Systèmes Thermiques" is a laboratory of Universities Paris VI and Paris XI, and CNRS (UMR 7608).

\section{REFERENCES}

[1] Pauchard L., Parisse F. and Allain C., Phys. Rev. E, 59 (1999) 3737.

[2] Allain C. and limat L., Phys. Rev. Lett., 74 (1995) 2981.

[3] Boeck T., Bahr H. A., Lampenscherf S. and Bahr U., Phys. Rev. B, 59 (1999) 1408.

[4] Groisman A. and Kaplan E., Europhys. Lett., 25 (1994) 415.

[5] Shorlin K. A., de Bruyn J. R., Graham M. and Morris S. W., Phys. Rev. E, 61 (2000) 6950.

[6] Annarelli C. C., Fornazero J., Bert J. and Colombani J., Eur. Phys. J. E, 5 (2001) 599.

[7] Crank J. and Park G. S., in Diffusion in Polymers (Academic Press) 1968.

[8] Parisse F. and Allain C., J. Phys. II, 6 (1996) 1111.

[9] Deegan R. D., Bakajin O., Dupont T. F., Huber G., Nager S. R. and Witten T., Nature, 389 (1997) 827.

[10] PlazeK D. J. and Ngai K. L., The glass temperature, in Physical Properties of Polymer Handbook, Part 12 (AIP Press) 1996.

[11] De Gennes P. G., Eur. Phys. J. E, 6 (2001) 421.

[12] Parisse F. and Allain C., Langmuir, 13 (1997) 3598.

[13] De Gennes P. G., in Scaling Concepts in Polymer Physics (Cornell University Press, Ithaca, New York) 1979.

[14] Vink H., Eur. Polym. J., 7 (1971) 1411.

[15] Güner A. and Kibarer G., Eur. Polym. J., 37 (2001) 619.

[16] Incropera F. P. and DE Witt D. P., in Fundamentals of Heat and Mass Transfer (John Wiley and Sons, New York) 1990.

[17] Dor M. and Edwards S. F., in The Theory of Polymer Dynamics (Clarendon Press, Oxford) 1986.

[18] Timoshenko S. and Gere J. M., in Theory of Elastic Stability (McGraw-Hill, New York) 1961, 2nd edition.

[19] Landau L. D. and Lifshitz E. M., in Theory of Elasticity (Pergamon Press, New York) 1959.

[20] Note that, in these calculations, the shell thickness, $h$, is supposed to be uniform, time independent and smaller than the other characteristic lengths involved in the problem. Moreover, $h$ is assumed to be small enough to consider that the length change due to the curvature inversion (the external curvature becomes an internal curvature) has a low contribution to the total elastic energy compared to the bending contribution.

[21] De Gennes P. G., Eur. Phys. J. E, 7 (2002) 31. 